\title{
Construction of Lanthanum Vanadate/Functionalized-Boron Nitride Nanocomposite: The Electrochemical Sensor for Monitoring of Furazolidone
}

Thangavelu Kokulnathan $^{\mathrm{a}, \mathrm{b}}$, Ghzzai Almutairi ${ }^{\mathrm{c}}$, Shen-Ming Chen ${ }^{\mathrm{a} *}$, Tse-Wei Chen ${ }^{\mathrm{a}, \mathrm{d}, \mathrm{e}}$, Faheem Ahmed $^{\mathrm{f}}$, Nishat Arshi $^{\mathrm{g}}$, Bandar AlOtaibi $^{\mathrm{c}}$

${ }^{a}$ Electroanalysis and Bioelectrochemistry` Lab, Department of Chemical Engineering and Biotechnology, National Taipei University of Technology, Taipei 10608, Taiwan.

${ }^{b}$ Department of Electro-Optical Engineering, National Taipei University of Technology, Taipei 10608, Taiwan.

${ }^{c}$ National Center for Energy Storage Technologies, King Abdulaziz City for Science and Technology (KACST), Riyadh 12354, Saudi Arabia.

${ }^{d}$ Research and Development Center for Smart Textile Technology, National Taipei University of Technology, Taipei 106, Taiwan.

${ }^{e}$ Department of Materials, Imperial College London, London, SW7 2AZ, United Kingdom.

${ }^{f}$ Department of Physics, College of Science, King Faisal University, P.O Box 400, Hofuf, Al-Ahsa 31982, Saudi Arabia.

${ }^{g}$ Department of Basic Sciences, Preparatory Year Deanship, King Faisal University, P.O Box 400, Hofuf, Al-Ahsa 31982, Saudi Arabia

Corresponding author

*E-mail: smchen78@ms 15.hinet.net

Tel: +8862270 17147, Fax: +886227025238.

Number of Pages 6

Number of Figures 5

Number of Table 1 


\begin{tabular}{lll}
\hline Entry & Table of Contents & Page. No \\
\hline & Materials, Reagents and Measurements. & S3 \\
\hline & Instruments & S3 \\
\hline & Functionalization of F-BN & S3 \\
\hline & Synthesis of LaV/F-BN nanocomposite & S3 \\
\hline Fig. $\mathbf{S 1}$ & TEM image of h-BN & $\mathbf{S 4}$ \\
\hline Fig. $\mathbf{S 2}$ & HAADF-STEM of LaV/F-BN nanocomposite & $\mathbf{S 4}$ \\
\hline Fig. $\mathbf{S 3}$ & BET & $\mathbf{S 5}$ \\
\hline Fig. $\mathbf{S 4}$ & CV signals of bare and modified electrodes in the 0.05M PB (pH 7.0) & $\mathbf{S 5}$ \\
& without FZD. & \\
\hline
\end{tabular}

Fig. S5 (A) Selectivity test of LaV/F-BN/RDGCE with the successive addition $\quad$ S6 of $100 \mu \mathrm{M}$ FZD (a) and higher concentration of interfering species (bs) in $0.05 \mathrm{M} \mathrm{PB}$ (pH 7.0). (B) The operational stability of the LaV/FBN/RDGCE.

Table S1 FZD detection in human blood serum and urine samples.

Materials, Reagents and Measurements.

Lanthanum (III) nitrate hexahydrate $\left(\mathrm{La}\left(\mathrm{NO}_{3}\right)_{3} \cdot 6 \mathrm{H}_{2} \mathrm{O}\right)$, ammonium metavanadate $\left(\mathrm{NH}_{4} \mathrm{VO}_{3}\right), \mathrm{BN}$, hydroquinone (HQ), ethanol and all other chemicals (including interfering species) were purchased from Sigma-Aldrich and used without further purification. All the solutions in the experiments were prepared with ultrapure double ionized (DI) water.

\section{Instruments}

Field emission scanning electron microscope (FESEM, ZEISS Sigma 300 microscope) and high-resolution transmission electron microscopy (HRTEM, Shimadzu JEM-1200 EX, 200 kV) were used to study the morphology of the as-prepared samples. The composition and crystal structures of the nanomaterials were analyzed by X-ray diffraction (XRD) on an XPERT-PRO diffractometer (PANalytical B.V., The Netherlands) with $\mathrm{Cu}-\mathrm{K} \alpha$ radiation $(\lambda=1.5406 \AA)$ in the $2 \theta$ scan range from $10^{\circ}$ to $90^{\circ}$. The chemical and surface electronic state of the nanomaterial was scrutinized by X-ray photoelectron spectroscopy (XPS; Thermo scientific multi-lab 2000). 
Electrochemical impedance spectroscopy (EIS) measurements were examined by using ZAHNER scientific instruments (THALES software package). Chemical composition of nanocomposite was performed on a Fourier transform infrared analysis (JASCO 6600, FT-IR) spectrophotometer. All the electrochemical measurements were performed on a CHI 750A Electrochemical workstation (CH Instruments (USA)) with a conventional three-electrode system composed of a platinum wire (Pt) as the auxiliary electrode, an $\mathrm{Ag} / \mathrm{AgCl}$ saturated $\mathrm{KCl}$ as reference electrode and a bare or modified glassy carbon working electrode/rotating disk GCE (GCE/ RDGCE), respectively. All the electrochemical experiments were carried out in a nitrogen atmosphere at room temperature.

\section{Functionalization of F-BN}

The F-BN was synthesized by a simple sonochemical approach in this work [61]. The hBN $(5 \mathrm{~g})$ and HQ $(0.1 \mathrm{M})$ were added to $50 \mathrm{~mL}$ of DI water by sonochemical method. The precursor was constantly sonicated at room temperature for $1 \mathrm{~h}$. Finally, a white product was collected by centrifugation and washed with DI water. Afterwards, the final sample (F-BN) was dried in an oven at $60^{\circ} \mathrm{C}$ for $12 \mathrm{~h}$.

\section{Synthesis of LaV/F-BN nanocomposite}

Briefly, $0.5 \mathrm{M}$ of $\mathrm{NH}_{4} \mathrm{VO}_{3}$ was dissolved in $50 \mathrm{~mL}$ of DI water and was stirred for $30 \mathrm{~min}$ at a certain temperature. Subsequently, $0.5 \mathrm{M}$ of $\mathrm{La}\left(\mathrm{NO}_{3}\right)_{3} \cdot 6 \mathrm{H}_{2} \mathrm{O}$ was added to the above solution. After stirring for a few minutes, the above mixture was transferred to a $100 \mathrm{~mL}$ Teflon lined autoclave and kept at $180^{\circ} \mathrm{C}$ for $12 \mathrm{~h}$. After the reaction, the obtained product (LaV) was rinsed several times with DI water/ethanol and collected by centrifugation. Finally, the LaV was dried and calcined at $400{ }^{\circ} \mathrm{C}$ for $2 \mathrm{~h}$. F-BN $(60 \%)$ and $\mathrm{LaV}(40 \%)$ were dispersed in DI water at $50^{\circ} \mathrm{C}$ and sonicated for 30 mins to form homogeneous ink. Then, LaV/F-BN nanocomposite was washed for few times and dried at $60^{\circ} \mathrm{C}$ overnight. 


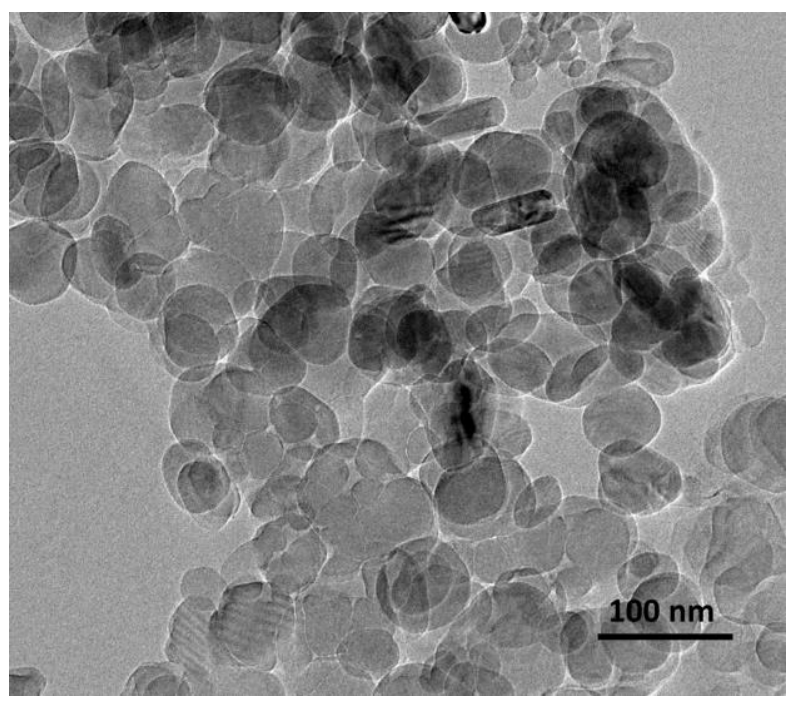

Fig. S1 TEM image of h-BN.

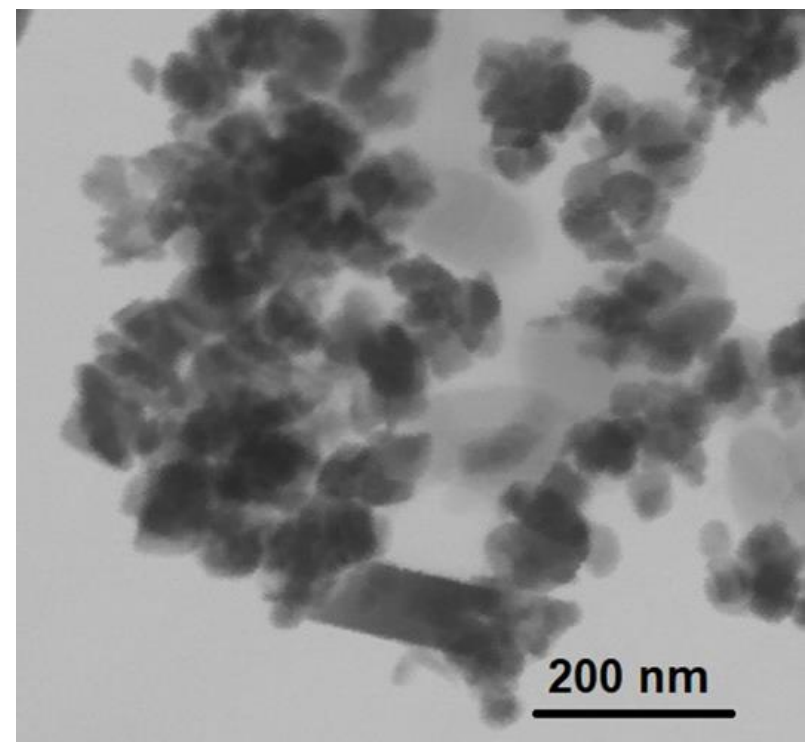

Fig. S2 HAADF-STEM of LaV/F-BN nanocomposite.

To understand the surface nature in terms of specific surface area BET analysis was carried out. The $\mathrm{N}_{2}$ - adsorption and desorption isotherms of the samples are given in Fig. S3. The isotherms obtained for all three samples could be assigned to type III isotherm, suggests the mesoporous nature of the samples. The measured specific BET surface area of F-BN, LaV and $\mathrm{LaV} / \mathrm{F}-\mathrm{BN}$ is $48.78,21.98$ and $30.56 \mathrm{~m}^{2} \mathrm{~g}^{-1}$ respectively. The high specific surface area of F-BN is due to the interspace between the irregular arrangement of F-BNs, which is in agreement with 
the TEM images. In comparison with $\mathrm{LaV}$, the larger surface area observed for the LaV/F-BN composite indicates the intra-space between the LaV and F-BN sheets.

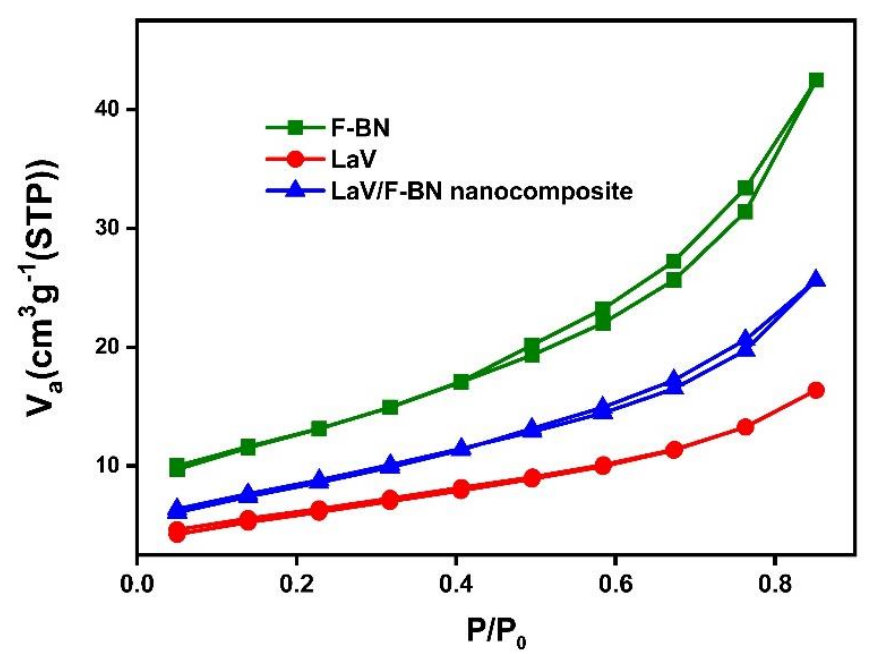

Fig. S3 BET $\mathrm{N}_{2}$ adsorption-desorption curves of LaV, F-BN and LaV/F-BN nanocomposite.

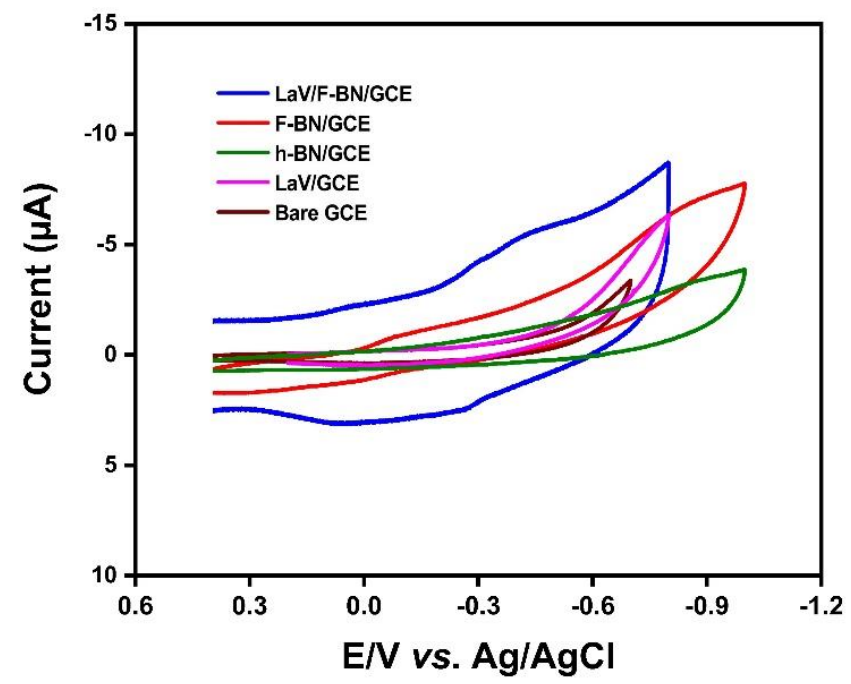

Fig. S4 CV signals of bare and modified electrodes in the $0.05 \mathrm{M} \mathrm{PB}(\mathrm{pH} 7.0)$ without FZD. 

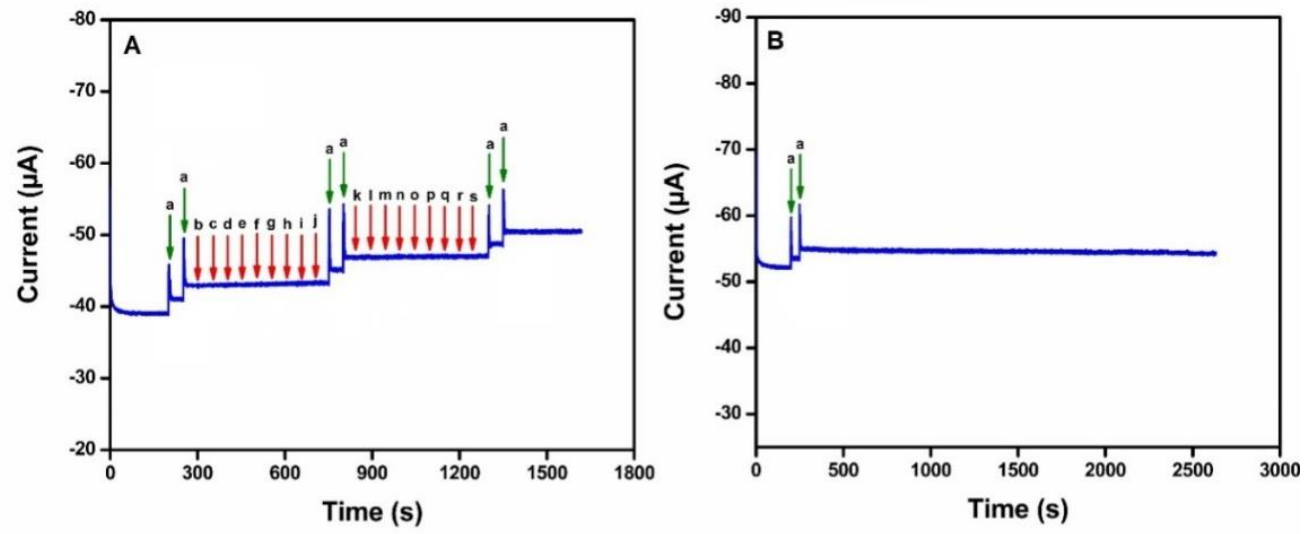

Fig. S5 (A) Selectivity test of LaV/F-BN/RDGCE with the successive addition of $100 \mu \mathrm{M}$ FZD (a) and higher concentration of interfering species (b-s) in $0.05 \mathrm{M} \mathrm{PB}(\mathrm{pH} 7.0)$. (B) The operational stability of the LaV/F-BN/RDGCE.

Table S1 FZD detection in human blood serum and urine samples.

\begin{tabular}{cccc}
\hline Samples & $\begin{array}{c}\text { FZD Added } \\
(\boldsymbol{\mu M})\end{array}$ & $\begin{array}{c}\text { FZD Found } \\
(\boldsymbol{\mu M})\end{array}$ & $\begin{array}{c}\text { Recovery } \\
(\boldsymbol{\%})\end{array}$ \\
\hline \multirow{3}{*}{ Human blood serum } & - & - & - \\
\cline { 2 - 4 } & 1.00 & 0.95 & 95.00 \\
\cline { 2 - 4 } & 2.00 & 1.97 & 98.50 \\
\cline { 2 - 4 } Human urine & 3.00 & 2.90 & 96.70 \\
\cline { 2 - 4 } & - & - & - \\
\cline { 2 - 4 } & 1.00 & 0.97 & 97.00 \\
\cline { 2 - 4 } & 2.00 & 1.95 & 97.50 \\
\hline & 3.00 & 2.95 & 98.30 \\
\hline
\end{tabular}

